\title{
National differences in screening programmes for cardiovascular risks could obstruct understanding of cardiovascular prevention studies in Europe
}

\author{
S. L. Thio • Th. B. Twickler • M. J. Cramer • P. Giral
}

Published online: 17 August 2011

(C) The Author(s) 2011. This article is published with open access at Springerlink.com

\begin{abstract}
Introduction In North-West Europe, cardiovascular disease is still a major cause of death and despite several efforts (e.g. European guidelines and conferences) cardiovascular risk factors are still inconsistently diagnosed and treated.

Methods We evaluated the first consultations of patients in two cardiovascular referral clinics in France and the Netherlands, while evaluating the differences in national guidelines and between the profiles of patients at their first consultation.

Results Notable differences exist between the two locally used guidelines in their programmes of cardiovascular risk assessment and their definition of LDL-cholesterol target levels. With regard to the LDL-cholesterol levels, more patients are 'on target' when using the French guideline than when using the Dutch guideline. Evaluation of the patient's profile at first presentation showed that the LDL-cholesterol levels were significantly lower in the Dutch patients $(n=77)$ compared with the French patients $(n=119)$. Dutch patients used significantly more statins than French patients.
\end{abstract}

\section{S. L. Thio $(\bowtie)$}

Medical Faculty, Leiden University Medical Centre,

Leiden, the Netherlands

e-mail: sl_thio@hotmail.com

T. B. Twickler

Department of Vascular Medicine, Academic Medical Centre,

University of Amsterdam,

Amsterdam, the Netherlands

M. J. Cramer

Department of Cardiology, University Medical Centre Utrecht,

Utrecht, the Netherlands

P. Giral

Department of Endocrinology and Metabolic Diseases,

La Pitié-Salpêtrière Hospital,

Paris, France
Conclusion Despite the small study population included in this study, we found that comparison of daily care (as part of a primary prevention programme) is rather difficult due to several national differences in the approach to patients. All these factors combined should be taken into account, when discussing and extrapolating results obtained from analysis of cardiovascular prevention programmes.

Keywords Primary prevention - Cardiovascular diseases . Practice guideline $\cdot$ Dyslipidemia

\section{Introduction}

In North-West Europe, cardiovascular disease is a major cause of death accounting for over 2 million deaths each year ( $42 \%$ of all mortality causes). Cardiovascular mortality is $55 \%$ in female and $43 \%$ in male subjects in Europe [1]. In daily care concerning cardiovascular prevention, a point of sincere concern, is the persistent manifestation of clinical inertia (knowing the presence of a risk factor but not acting appropriately with an intervention). The EuroAspire initiative evidently showed that patients with various established cardiovascular risk factors are still insufficiently treated and that this suboptimal treatment continues to exist, despite the existence of global clinical guidelines [2]. Comparing EuroAspire I (1997) with EuroAspire III (2009), the proportion of undertreated dyslipidaemia appears to be $80 \%$ and $51 \%$, respectively [2,3]. Against this background, several programmes have been started in Europe with the aims to improve screening for cardiovascular risk factors in at-risk populations.

Taking this knowledge into consideration, we evaluated the current approach in two cardiovascular clinics in NorthWest Europe (the Academic Medical Centre (AMC) of the 
University of Amsterdam in Amsterdam, the Netherlands and La Pitié-Salpêtrière in Paris, France). In the Netherlands and in France, cardiovascular disease accounts for $30 \%$ and $28 \%$, respectively, of the total mortality [4, 5], making it a prominent factor in daily health care. In both countries cardiovascular clinics, as part of cardiovascular prevention programmes, have been evolving in the last decade and are supported by the development of national and European clinical guidelines. Differences in health care approach may be of importance when comparing results from each region.

In this study we evaluated the content of the first consultation of patients with primary dyslipidaemia as part of cardiovascular prevention programmes that are currently running in the two cardiovascular clinics in Amsterdam and Paris.

\section{Methods}

Design

A retrospective analysis with a cross-sectional design was used. All patients referred for a specialised cardiovascular risk assessment to the vascular clinic of the AMC and the Hopital de Jour de Prevention Cardiovasculaire of the La Pitié-Salpêtrière Hospital were eligible for our analysis. These patients were referred by their general practitioner and were already using lipid-lowering medication. Cases were randomly selected from the total patient population visiting these clinics from May 2008 to April 2009.

\section{Patient selection}

Patients with ages between 35 and 75 years, who were treated in a primary prevention setting and were using lipidlowering drugs, were eligible for inclusion. Patients with comorbidity (such as an established type 2 or type 1 diabetes mellitus or a thyroid or liver disease) were excluded from the analysis, as these are confounders for primary dyslipidaemia.

In the Netherlands and in France a total of 77 and 119 patients were included, respectively. From each patient anthropometrics and direct data were collected, such as age, gender, weight, height, blood pressure, smoking habits, alcohol consumption and current lipid-lowering treatments. Venous blood samples in fasting state were drawn to assess lipid profile.

Description clinical programme's environment

Information about the structure of each distinctive cardiovascular clinic was collected and observed while visiting each specific centre. In the Amsterdam clinic, the guideline of the Centraal BegeleidingsOrgaan (CBO, a governmental institute for the enhancement of health care quality) on cardiovascular risk management was used [6] and in the Paris clinic, the HAS (Haute Autorité de Santé) recommendations on dyslipidaemia were used [7].

Statistical analysis methods

The collected data were processed into an SPSS file. Descriptive statistics and independent non-parametric tests were used to evaluate differences between the French and Dutch populations. A p-value below 0.05 (two-side tested) was considered as statistically significant. SPSS software, version 16 , was used.

\section{Results}

Comparison of infrastructures in a French and a Dutch cardiovascular clinic

The infrastructure of the cardiovascular clinics in which patients are assessed is different. A schematic overview is shown in Fig. 1.

The cardiovascular clinic of the La Pitié-Salpêtrière Hospital is a specialised unit in which structured evaluations of individual cardiovascular risk profiles are performed. This structure was described previously in detail [8]. In short, the one-day programme is guided by a strict protocol that consists of distinctive stations in a carousel-like structure. The vascular outpatient clinic in the AMC has a structured protocol to include patients in their cardiovascular prevention programme, which expands over a longer time period. After they are registered, patients are planned for a consultation appointment that will take place within one month's time. Results of blood analyses will be available at their arrival for the first consultation. After evaluation of the individual patient's results, a therapeutic strategy will be determined. The aims are discussed with the patient and the results will be evaluated in a regular follow-up.

\section{Comparison of French and Dutch guidelines}

Comparing the distinctive guidelines, it appears that they are rather uniform in both their approach of patients and their interventions. One major difference between French and Dutch guidelines is the calculation of patients' individualised risk of the occurrence of an ischaemic event in a timeframe of 10 years. The Dutch guideline implements the SCORE table [9]. With use of the 10-year mortality risk and other individual factors (as family history, 
Fig. 1 Flow diagram of clinic protocols in France (left) versus the Netherlands (right).

Schematic representation of the infrastructure used in the cardiovascular clinic in the Netherlands (Lipid Outpatient Clinic, Academic Medical Centre, Amsterdam) and in France (Hôpital de Jour de Prevention Cardiovasculaire, La Pitié-Salpêtrière hospital, Paris), showing the steps each patient is to take

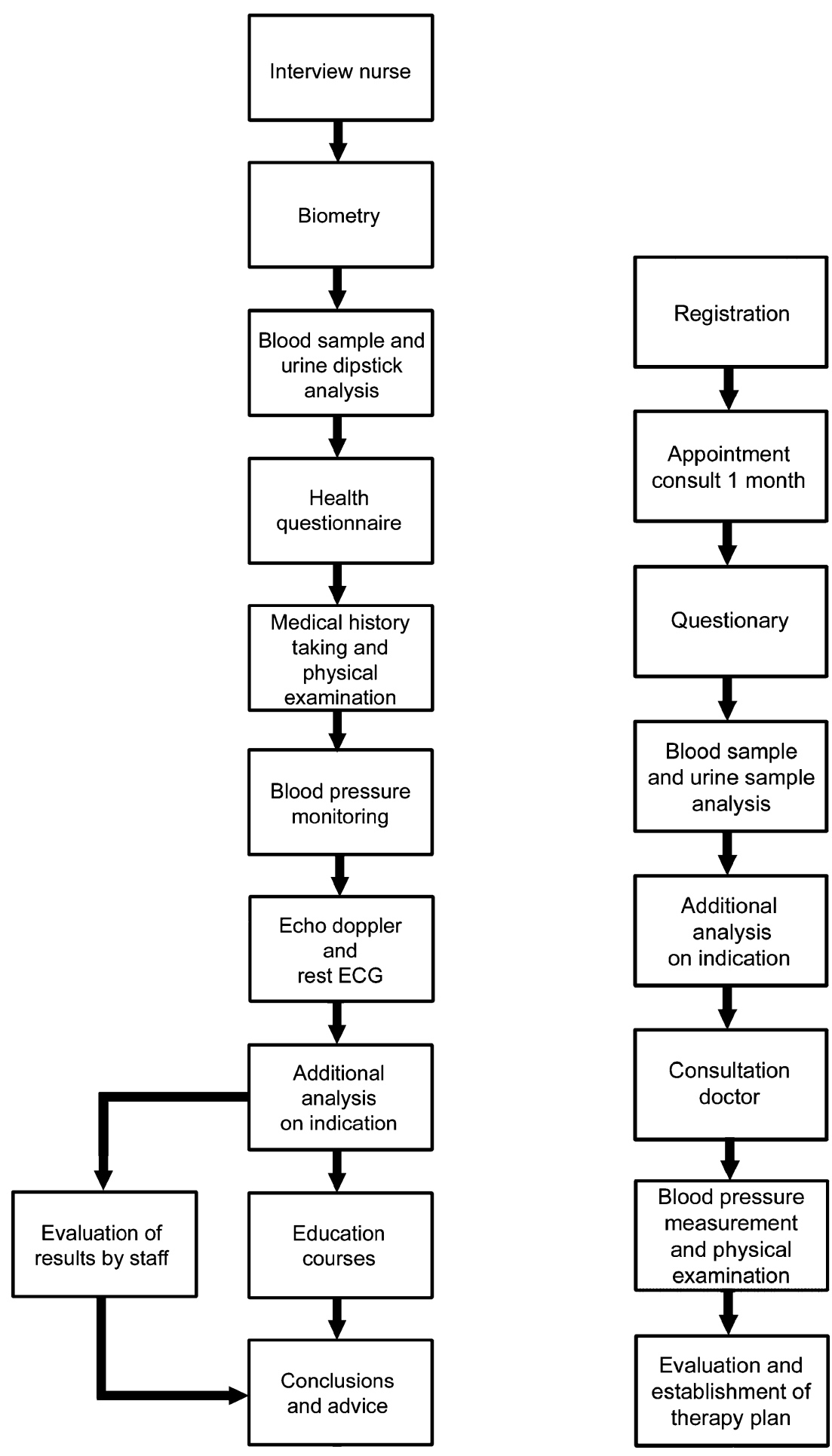

nutritional habits, alcohol intake and physical activity), an individual global risk profile is defined to establish an intervention strategy. Risk estimation in France is done according to the number of risk factors present, which will determine its treatment targets. Another difference between the two guidelines is the target level of LDL cholesterol. The Dutch guideline aims to achieve LDL-cholesterol levels lower than $2.5 \mathrm{mmol} / 1$, or a decrease of at least $1 \mathrm{mmol} / \mathrm{L}$ for all patients with a 10 -year risk of more than $10 \%$. In France, however, distinctive targets regarding LDL-cholesterol levels have been defined according to the number of risk factors present; LDL-cholesterol levels lower than $2.5 \mathrm{mmol} / \mathrm{L}$ are only pursued in secondary prevention programmes. 
Patient characteristics at first consultation in the two cardiovascular clinics

In the Dutch cardiovascular clinic 77 patients were documented and in France a total of 119 patients. Patients' characteristics are depicted in Table 1. Dutch patients present to the cardiovascular clinic at younger ages compared with French patients $(p<0.001)$. In both countries male patients tend to be referred at a younger age than female patients. This finding was not, however, statistically significant.

Lipid profiles are listed in Table 2. Lipid levels in Dutch patients seem to be lower than in patients visiting the French lipid clinic, but a significant difference was only ascertained for LDL cholesterol. The averages of absolute LDL-cholesterol levels were $3.1 \mathrm{mmol} / \mathrm{L}$ and $3.5 \mathrm{mmol} / \mathrm{L}$ for patients in the Netherlands and in France respectively.

When arranging the two groups according to gender, significantly lower levels of total cholesterol $(p=0.01)$, LDL cholesterol $(p=0.009)$ and HDL cholesterol $(p=0.02)$ were found in Dutch male patients compared with French male patients. Considering the female patients, a significant difference was only found in HDL-cholesterol levels ( $p=$ 0.003), with French females having higher levels.

The lipid-lowering medication that patients used at their first consultation was evaluated. Dutch patients use significantly more statins than French patients: 98\% versus $75 \%$ in male patients $(p=0.002)$ and $97 \%$ versus $83 \%$ in female patients $(p=0.04)$ respectively. Considering the different compounds in the statin group, Dutch patients use significantly more rosuvastatin than French patients $(p=$ 0.001). Furthermore, ezetimibe is used more often in Dutch patients, with $32 \%$ versus $21 \%$ in Dutch and French male patients, respectively, $(p=0.18)$ and $36 \%$ versus $15 \%$ in
Dutch and French female patients, respectively, $(p=0.03)$. French patients tend to use more fibrates (not significant).

In Table 3 we show the number of patients that are 'on LDL-cholesterol target' using either the Dutch or French guideline. More Dutch patients are 'on target', according to both the Dutch and the French guidelines. Considering Dutch target levels, $29 \%$ of Dutch patients and $17 \%$ of French patients are 'on target'. According to French target levels, $87 \%$ of Dutch patients and $80 \%$ of French patients are 'on target'. Regarding differences between genders, more male patients are 'on target' compared with female patients in the Dutch group, whereas in the French group the number of patients 'on target' is more evenly distributed among men and women.

\section{Discussion}

Despite recent efforts to develop general European guidelines, remarkable differences are found between currently running cardiovascular prevention programmes in two cardiovascular clinics in North-West Europe. This observation will warn for extrapolations from national analysis of cardiovascular studies performed within the European Union. Moreover, this fact may limit accurate comparisons regarding the cardiovascular health status among European countries and restrict collaborative therapeutic goals.

In cardiovascular prevention programmes several European initiatives (such as the EuroAspire programme) have been undertaken with the aim of establishing uniform clinical approaches to patients and providing transparency of results and subsequent treatments. The two cardiovascular centres that we have compared use national guidelines that are based on evidence derived from international

Table 1 Anthropometric characteristics (age, body mass index, systolic and diastolic blood pressure, smoking, use of alcohol and positive family history) of the research population at their first consultation, specified for country and gender

\begin{tabular}{|c|c|c|c|c|c|c|}
\hline \multirow[t]{2}{*}{ Variable } & \multicolumn{3}{|l|}{ Netherlands } & \multicolumn{3}{|l|}{ France } \\
\hline & All $(n=77)$ & Male $(n=41)$ & Female $(n=36)$ & All $(n=119)$ & Male $(n=73)$ & Female $(n=46)$ \\
\hline \multicolumn{7}{|l|}{ Mean (SD) } \\
\hline Age & $52.2 *(9.1)$ & $49.4(8.5)$ & $55.3(8.7)$ & $58.3 *(10.3)$ & $56.5(10.0)$ & $62.5(9.6)$ \\
\hline BMI & $26.8(3.5)$ & $27.0(2.9)$ & $26.7(4.1)$ & $26.1(3.7)$ & $26.1(3.1)$ & $26.0(4.6)$ \\
\hline SBP (mmHg) & $129.6^{*}(15.0)$ & $120.0(14.7)$ & $129.1(15.5)$ & $125.0 *(15.0)$ & $125.4(13.5)$ & $124.3(17.2)$ \\
\hline $\mathrm{DBP}(\mathrm{mmHg})$ & $79.7 \dagger(8.6)$ & $80.1(8.2)$ & $79.2(9.1)$ & $71.0 \dagger(8.8)$ & $73.7(8.3)$ & $66.5(7.6)$ \\
\hline \multicolumn{7}{|l|}{ Number (\%) } \\
\hline Smokers & $16(20.8)$ & $11(26.8)$ & $5(13.9)$ & $22(18.5)$ & $18(24.7)$ & $4(8.7)$ \\
\hline Alcohol & $26(33.8)$ & $13(31.7)$ & $13(36.1)$ & $37(31.1)$ & $29(39.7)$ & 9 (19.6) \\
\hline Positive family & $48(62.3)$ & $25(61.0)$ & $23(63.9)$ & $61(51.3)$ & $38(52.1)$ & $23(50.0)$ \\
\hline
\end{tabular}

$B M I$ body mass index; $S B P$ systolic blood pressure; $D B P$ diastolic blood pressure; alcohol $\geq 1$ unit/day; positive family is familiar hypercholesterolaemia or cardiovascular event age $<55$ in first-degree male relatives, age $<65$ in first-degree female relatives

${ }^{*} p<0.05, \dagger p<0.01$ 
Table 2 Levels of serum lipids (total cholesterol, LDL-cholesterol, HDL-cholesterol and triglycerides) of the patients at their first consultation, specified for country and gender

\begin{tabular}{|c|c|c|c|c|c|c|}
\hline \multirow[t]{2}{*}{ Variable } & \multicolumn{3}{|l|}{ Netherlands } & \multicolumn{3}{|l|}{ France } \\
\hline & All $(n=77)$ & Male $(n=41)$ & Female $(n=36)$ & All $(n=119)$ & Male $(n=73)$ & Female $(n=46)$ \\
\hline \multicolumn{7}{|l|}{ Mean (SD) } \\
\hline Total cholesterol $(\mathrm{mmol} / \mathrm{L})$ & $5.2(1.2)$ & $4.9 *(1.1)$ & $5.5(1.9)$ & $5.5(1.4)$ & $5.3^{*}(1.2)$ & $5.8(1.7)$ \\
\hline LDL cholesterol (mmol/L) & $3.1 *(1.0)$ & $2.9 \dagger(0.9)$ & $3.4(1.1)$ & $3.5^{*}(1.6)$ & $3.5 \dagger(1.1)$ & $3.6(1.2)$ \\
\hline HDL cholesterol $(\mathrm{mmol} / \mathrm{L})$ & $1.4(0.4)$ & $1.2 *(0.3)$ & $1.5 \dagger(0.4)$ & $1.3(0.6)$ & $1.4 *(0.6)$ & $1.2 \dagger(0.5)$ \\
\hline Triglycerides $(\mathrm{mmol} / \mathrm{L})$ & $1.7(1.5)$ & $1.8(1.4)$ & $1.5(1.5)$ & $1.5(1.0)$ & $1.5(0.6)$ & $1.5(1.1)$ \\
\hline
\end{tabular}

$L D L$ low density lipoprotein; $H D L$ high density lipoprotein

$* p<0.05, \dagger p<0.01$

available resources. This presumes that a certain degree of uniformity in treatment and clinical results regarding cardiovascular risk factors would be present.

In this report we have shown that notable differences nevertheless do exist in clinical approaches and subsequently in the number of patients 'on target'. We observed two marked differences between the nationally used guidelines: firstly in methods of cardiovascular risk assessment and secondly the target level of LDL cholesterol. The less rigid strategy of the French guideline towards LDLcholesterol target levels results in more patients being considered 'on target' at their first presentation, compared with patients assessed according to the Dutch guideline. We also observed that the differences in target levels of LDL cholesterol lead to a different clinical approach regarding lipid-lowering strategies. Indeed, lipid profiles differ in the two patient groups at their first visit. These results are in accordance with prior literature: different assessments of cardiovascular risk factors will result in different risk classifications (patients in a low- or a high-risk group) and subsequently a different treatment strategy [10].

Following these observations, we found that a difference in the proportion of patients that achieved their treatment targets was present when applying either the Dutch or the French guideline. The proportion of patients 'on target' at their first visit is significantly different when patients are scored with the French guideline or the Dutch guideline.
Taking this into consideration, different reports on achieving treatment targets regarding cardiovascular prevention would be the outcome depending on which guideline is used. When comparing EuroAspire I and II with EuroAspire III [2, 3, 11], the number of patients 'on target' (according to guidelines developed by Joint European Societies) remains 50\% despite more awareness of cardiovascular prevention and increased use of statins during the observation period. In these evaluations, the number of patients 'on target' ranges from $30 \%$ to $75 \%$ between distinctive European countries. From our results we did indeed find the same range in the number of patients 'on treatment target' according to each national guideline (29\% versus $87 \%$ in Dutch versus French guidelines, respectively). Our observations strongly support that, despite the presence of evidence-based European guidelines (ESC guidelines [12]), considerable differences still exist in cardiovascular risk management between two European referral centres. This finding may limit interpretation of other European multicentre studies in which local guidelines are applied for distinctive subpopulations. It is of importance to notice that marked differences exist in treatment strategies and patient profiles at their first consultation.

In conclusion, when evaluating programmes for primary prevention of cardiovascular disease in two cardiovascular clinics in North-West Europe, differences in currently used guidelines are present and this could lead to a different

Table 3 Proportion of patients that are 'on target', when using either the Dutch or the French guideline, assessed on both research populations

\begin{tabular}{|c|c|c|c|c|c|c|}
\hline \multirow[t]{2}{*}{ Variable } & \multicolumn{3}{|l|}{ Netherlands } & \multicolumn{3}{|l|}{ France } \\
\hline & All $(n=77)$ & Male $(n=41)$ & Female $(n=36)$ & All $(n=119)$ & Male $(n=73)$ & Female $(n=46)$ \\
\hline \multicolumn{7}{|l|}{ Number (\%) } \\
\hline Dutch guideline on target & $22(29)$ & $14(34)$ & $9(25)$ & $20(17)$ & $12(16)$ & $9(20)$ \\
\hline French guideline on target & $67(87)$ & $37(90)$ & $30(83)$ & $95(80)$ & $59(81)$ & $36(78)$ \\
\hline
\end{tabular}

Dutch guideline LDL target $<2.5 \mathrm{mmol} / \mathrm{L}$; French guideline LDL target according to number of risk factors 
approach in health care. Despite these differences it is not known whether this will result in a less beneficial outcome during follow-up. Uniformity is becoming an important issue in current daily health care. Our observations show that, despite progress in developing European guidelines (such as the ESC guidelines), more European initiatives that will also highlight gender-related differences should be launched.

Acknowledgements Sioe Lie Thio is a medical student at Leiden University, who visited the Cardiovascular Prevention Clinic in l'Hôpital La Pitié-Salpêtrière in Paris. The French-Dutch Network (Anièce Heijnen) and the Curators Fund Leiden are gratefully thanked for their financial support. Dr Philippe Giral and other members of the Endocrinology Department in l'Hôpital La Pitié-Salpêtrière are also thanked for their warm hospitality. And finally Dr Marcel Twickler is sincerely thanked for his enthusiastic support and all his advice for the achievement of this article.

Open Access This article is distributed under the terms of the Creative Commons Attribution Noncommercial License which permits any noncommercial use, distribution, and reproduction in any medium, provided the original author(s) and source are credited.

\section{References}

1. Allender S, Scarborough P, Peto V, et al. European Cardiovascular Disease Statistics. 2008

2. EUROASPIRE. A European Society of Cardiology survey of secondary prevention of coronary heart disease: principal results. EUROASPIRE Study Group. European Action on Secondary
Prevention through Intervention to Reduce Events. Eur Heart J. 1997;18(10):1569-82.

3. Kotseva K, Wood D, De Backer G, et al. EUROASPIRE III: a survey on the lifestyle, risk factors and use of cardioprotective drug therapies in coronary patients from 22 European countries. Eur J Cardiovasc Prev Rehabil. 2009;16(2):121-37.

4. Statistics Netherlands 2008, www.cbs.nl.

5. French National Institute of Statistics and Economic Studies.

6. CBO Multidisciplinaire richtlijn Cardiovasculair risicomanagement 2006. 2006. Utrecht: Kwaliteitsinstituut voor de Gezondheidszorg CBO.

7. Haute Autorité de Santé (HAS). Les recommandations 2005 sur la prise en charge des dyslipidémies.

8. Timmerman MK, Rensing KL, Giral P, et al. Lessons from France: the cardiovascular prevention clinic in the la Pitie-Salpetriere Hospital in Paris. Neth Heart J. 2007;15(1):22-6.

9. Conroy RM, Pyorala K, Fitzgerald AP, et al. Estimation of ten-year risk of fatal cardiovascular disease in Europe: the SCORE project. Eur Heart J. 2003;24(11):987-1003.

10. Fornasini M, Brotons C, Sellares J, et al. Consequences of using different methods to assess cardiovascular risk in primary care. Fam Pract. 2006;23(1):28-33.

11. Lifestyle and risk factor management and use of drug therapies in coronary patients from 15 countries; principal results from EUROASPIRE II Euro Heart Survey Programme. Eur Heart J 2001;22(7):554-72.

12. Graham I, Atar D, Borch-Johnsen K, et al. European guidelines on cardiovascular disease prevention in clinical practice: full text. Fourth Joint Task Force of the European Society of Cardiology and other societies on cardiovascular disease prevention in clinical practice (constituted by representatives of nine societies and by invited experts). Eur J Cardiovasc Prev Rehabil. 2007;14 Suppl 2: S1-113. 